\title{
Encephalocele development from a congenital meningocele: case report
}

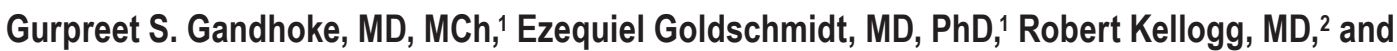 \\ Stephanie Greene, MD'
}

\author{
${ }^{1}$ Children's Hospital of Pittsburgh, University of Pittsburgh Medical Center, Pittsburgh, Pennsylvania; and 2Advocate Children's \\ Hospital, Park Ridge, Illinois
}

\begin{abstract}
A fetal MRI study obtained at 21 weeks' gestation revealed a suboccipital meningocele without hydrocephalus. One day after term birth, MRI demonstrated an acquired cerebellar encephalocele, and MRI obtained 5 months later showed progressive enlargement of the encephalocele, still without obvious hydrocephalus. The patient underwent an operation in which an external ventricular drain was placed, the grossly normal cerebellum was reduced into the posterior fossa without resection, and the dural defect was closed. The drain was weaned out over 5 days, and no ventriculoperitoneal shunt was placed. Postoperative MR images revealed normal cerebellum and no hydrocephalus. The patient is developmentally normal. Meningocele and encephalocele are embryologically distinct. An acquired encephalocele could develop from hydrocephalus (which was not present in this case), or secondary to the lower resistance to expansion into the dural defect of the meningocele relative to the resistance to expansion of the fetal skull. The cerebellar tissue was normal in this case, and was thus preserved. The developmental prognosis is excellent. To the authors' knowledge, this is the first reported case of this occurrence. It is important to differentiate between congenital and acquired encephalocele etiologies, because resection of the cerebellar tissue in an acquired encephalocele (as is routinely done in cases of congenital encephalocele) would be expected to result in neurological deficits.
\end{abstract}

https://thejns.org/doi/abs/10.3171/2017.6.PEDS17178

KEY WORDS meningocele; encephalocele; paraxial mesoderm; congenital

A $\mathrm{N}$ encephalocele is a protrusion of the CNS elements through a defect in the dura mater and cranium. The prevalence of encephalocele ranges from 0.08 to 0.5 per 1000 live births. ${ }^{1,7,10,12}$ Occipital encephaloceles are far more common in the Western world, whereas frontal or sincipital encephaloceles are more common in Asia. ${ }^{3,10}$ Only approximately $50 \%$ of fetuses with encephaloceles survive until birth, with the deaths occurring almost exclusively in fetuses with occipital encephaloceles. ${ }^{6,7}$ Most surviving infants with encephaloceles have cognitive deficits, and hydrocephalus, spasticity, and seizures are also common in these children. ${ }^{3,5,7}$ Up to half of these children have chromosomal abnormalities. In contrast, children with meningoceles, which do not contain neural tissue but only the leptomeninges, are neurologically normal, without an increased incidence of seizures or spasticity. ${ }^{10}$ As many as $65 \%$ of occipital meningoceles and encephaloceles are associated with hydrocephalus. ${ }^{4,10}$ Both encephaloceles and meningoceles are believed to develop as sequelae of failure of the paraxial mesoderm of the future dura and skull to fuse in the midline secondary to adhesion formation between the neural and cutaneous ectoderm prior to disjunction. ${ }^{8}$ To our knowledge, no case of an in utero meningocele becoming an encephalocele by the time of delivery has been previously reported.

\section{Case Report}

History

This female infant was born at full term with a prenatally observed suboccipital meningocele diagnosed on MRI studies obtained at 21 weeks' gestation (Fig. 1A 

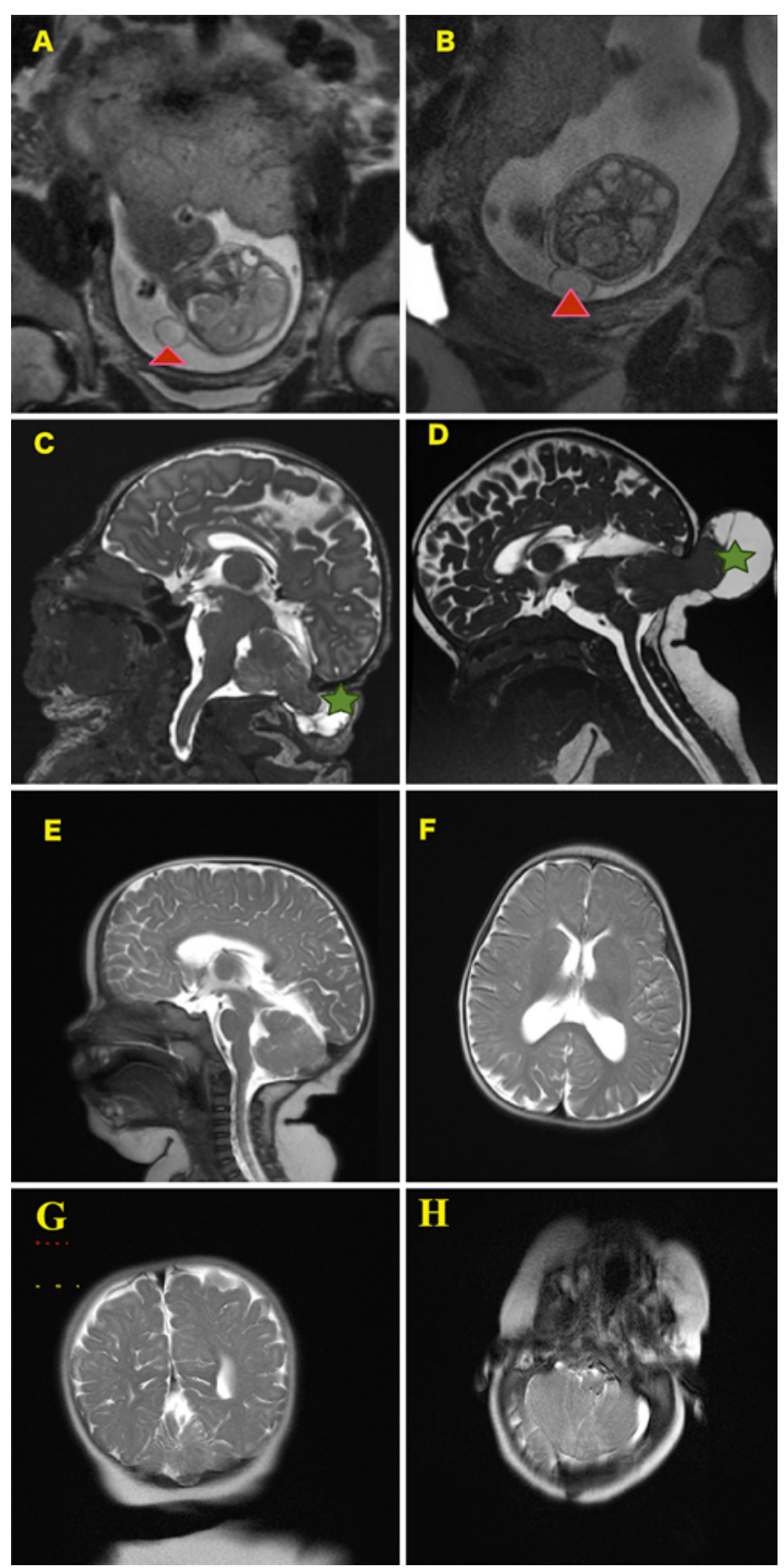

FIG. 1. A: Sagittal prenatal MRI view of suboccipital meningocele at 21 weeks' gestation. Arrowhead points to the meningocele. B: Axial prenatal MRI view of suboccipital meningocele at 21 weeks' gestation. Arrowhead points to the meningocele. C: Sagittal MRI view at birth revealing that the meningocele now has cerebellar tissue within it and no evidence of hydrocephalus. Star marks the herniated cerebellum into the prior meningocele. D: Sagittal MRI view at 5 months after birth showing progressive enlargement of the encephalocele, and no obvious hydrocephalus. Star marks the enlarging herniated cerebellum into the prior meningocele. E: One-year postoperative sagittal MRI view revealing no hydrocephalus, with normal subarachnoid spaces around the cerebellum and the craniovertebral junction. F: One-year postoperative axial MRI view revealing no hydrocephalus, with normal subarachnoid spaces around the cerebellum and the craniovertebral junction. G: One-year postoperative coronal MRI view revealing complete resolution of the encephalocele. H: One-year postoperative axial MRI view demonstrating complete resolution of the encephalocele. Figure is available in color online only. and B). A repeat MRI sequence obtained following birth revealed that the meningocele now had cerebellar tissue within it (Fig. 1C). There were no other intracranial anomalies. The infant had no evidence of hydrocephalus. The overlying skin was intact, although hairless. An MRI study obtained 5 months after birth showed progressive enlargement of the encephalocele, still without obvious hydrocephalus (Fig. 1D). The patient's parents noted that the occipital swelling changed in size with pressure and activity, and during the day.

\section{Examination}

The child was eating and gaining weight well. She had a social smile. She tracked movement visually and could support her head independently. With a length of $57 \mathrm{~cm}$, a weight of $5.9 \mathrm{~kg}$, and a head circumference of $40.5 \mathrm{~cm}$, she was at the 75th percentile for her age. She had a soft, $4-\mathrm{cm}$, suboccipital, hairless meningoencephalocele. Her anterior fontanelle was open, soft, and slightly sunken. Her pupils were equal and reactive to light. She tracked movement well visually. She had no setting sun sign. Her extraocular movements were full, and she had normal tone in all 4 extremities. At 5 months of age she had gained enough weight to tolerate the expected blood loss of surgery, and so plans were made for elective repair of the meningoencephalocele.

\section{Operation}

After placing a right frontal external ventricular drain, a linear incision was made over the meningoencephalocele. The encephalocele appeared to contain relatively normal cerebellar tissue, in contrast to the scarred, dysplastic tissue usually encountered in an encephalocele repair. The bony margin surrounding the dural defect was defined with electrocautery and a curette, and measured $3 \times 3 \mathrm{~cm}$. The dural margin was separated from the encephalocele with a Penfield No. 4 instrument, microscissors, and bipolar cautery. The encephalocele retracted within the skull after all adhesions to the dura were dissected. There was a significant egress of CSF from the left margin of the encephalocele after it retracted. The encephalocele was completely reduced within the posterior fossa with gentle pressure. There was adequate dura to close primarily without a patch. An autologous switch cranioplasty was performed. The excess skin was trimmed, and the galea and skin were reapproximated with absorbable suture.

\section{Postoperative Course}

The external ventricular drain was left in place for 5 days to allow for dural healing without tension. The drain was weaned out, and serial imaging demonstrated the absence of hydrocephalus during this process. The child was neurologically unchanged from her preoperative state. One year postoperatively she has no neurological impairment, and her incision has completely healed. An MRI study revealed no hydrocephalus, with normal subarachnoid spaces around the cerebellum and the craniovertebral junction (Fig. 1E and F). Coronal (Fig. 1G) and axial (Fig. $1 \mathrm{H})$ views demonstrate good retraction of the encephalocele in the posterior fossa. 


\section{Discussion}

No prior case of a prenatally diagnosed meningocele becoming an encephalocele by the time of delivery has been reported. Such a phenomenon may occur earlier in the gestation period than can be identified with current imaging modalities, but this case occurred late enough to be readily identifiable. Congenital encephaloceles classically contain gliotic neural tissue on pathological analysis; this tissue is routinely resected without neurological sequelae to close the dura and repair the skull defect. In this case of acquired encephalocele, the cerebellar tissue within the defect appeared grossly normal, without the firm, rubbery consistency typically encountered with congenital encephaloceles.

It has been postulated that encephaloceles are a form of paraxial mesoderm insufficiency in the developing fetus. ${ }^{8}$ Failure of this closure results in the typically midline location of most encephaloceles. The occipital location is the most common one in the US. Predictors of prognosis include the presence of brain tissue within the sac, microcephaly, size and site of the lesion, and the operability of the lesion. Classically, children with encephaloceles have significant rates of spastic paraparesis (11\%), moderateto-severe developmental delay (7\%), and hydrocephalus $(20 \%-65 \%){ }^{9,10}$ In contrast, our patient, with the delayed development of an encephalocele, is developmentally normal.

Hanley et al., ${ }^{5}$ using ultrasound, reported complete retraction of the occipital cortex from within an occipital encephalocele into the confines of the skull, between 27 and 35 weeks of gestation. They postulated either a retraction or thinning of the occipital cortex to explain this phenomenon. An important requirement for the development of an encephalocele is a bony defect in the skull. Cranial ossification begins at approximately 10 weeks of gestation, and thus diagnosing an encephalocele before that age is not possible. Bronshtein and Zimmer, ${ }^{2}$ using transvaginal ultrasound, described a case of an occipital encephalocele as early as 13 weeks of gestation. The lesion was $8 \times 9 \mathrm{~mm}$ and empty to begin with, and then 1 week later was filling with brain tissue. Repeated examinations at 15 and 16 weeks revealed complete resolution of the defect, and the maternal serum alpha protein level was normal. At 19 weeks, there was recurrence of the encephalocele, which persisted until termination of the pregnancy at 24 weeks of gestation. Van Zalen-Sprock et al., ${ }^{11}$ using transvaginal ultrasound, described a fetus at 11 weeks of gestation with 2 translucent areas in the occipital region. A repeat scan at 13 weeks demonstrated a bony defect and protrusion of the brain. The authors made the diagnosis of occipital encephalocele and confirmed this by pathological examination after termination of the pregnancy.

All 3 of these cases were limited by the imaging techniques available in the early 1990s, and so it is difficult to draw conclusions about the accuracy of the descriptions regarding the presence or absence of brain tissue within these dural defects. Our patient underwent fetal MRI and postnatal MRI, so the diagnoses of meningocele and subsequent encephalocele are much more certain. In our case, the antenatal MRI revealed a meningocele at 21 weeks of gestation. The MRI at birth revealed cerebellar tissue herniating into the meningocele. A repeat MRI sequence obtained at 6 months of age revealed further enlargement of the encephalocele.

The natural history of an encephalocele may not follow a set path. These defects should be serially imaged once diagnosed. The critical time to identify a defect is at 10 weeks, when ossification of the skull is beginning. Thereafter, following these lesions for their resolution or progressive incorporation of brain tissue is critical for prognostication. It seems that the herniation of brain tissue later in gestation may portend a better neurological prognosis; our patient remains developmentally normal, without seizures, microcephaly, or hydrocephalus. We postulate that the growing brain pushed the cerebellum out through the dural defect, following the path of least resistance into the meningocele. Once a part of the cerebellum herniated into the meningocele, the adhesions with the dural margin created a ball valve phenomenon, which led to a progressive increase in the size of the lesion, with the sac dilating secondary to the pulsatile CSF egress. This theory is reinforced by the fact that at surgery, once the adhesions between the herniated cerebellum and dura were dissected, we noted a gush of CSF from the neck of the encephalocele. Better understanding of the natural history of encephaloceles and their low prevalence begs for the reporting of such cases with serial MRI examinations, to better inform our patients' families regarding prognosis. Our report merits inclusion in the sparse literature available for this condition.

\section{Conclusions}

Families grappling with the diagnosis of an encephalocele should be informed of the potentially more favorable prognosis associated with later encephalocele formation, along with the favorable factors such as normocephaly and an operable location of the defect.

\section{References}

1. Adetiloye VA, Dare FO, Oyelami OA: A ten-year review of encephalocele in a teaching hospital. Int J Gynaecol Obstet 41:241-249, 1993

2. Bronshtein M, Zimmer EZ: Transvaginal sonographic follow-up on the formation of fetal cephalocele at 13-19 weeks' gestation. Obstet Gynecol 78:528-530, 1991

3. Chapman PH, Swearingen B, Caviness VS: Subtorcular occipital encephaloceles. Anatomical considerations relevant to operative management. J Neurosurg 71:375-381, 1989

4. Guthkelch AN: Occipital cranium bifidum. Arch Dis Child 45:104-109, 1970

5. Hanley ML, Guzman ER, Vintzileos AM, Leiman S, Doyle A, Shen-Schwarz S: Prenatal ultrasonographic detection of regression of an encephalocele. J Ultrasound Med 15:71-74, 1996

6. Lorber J: The prognosis of occipital encephalocele. Dev Med Child Neurol Suppl 13:75-86, 1967

7. Lorber J, Schofield JK: The prognosis of occipital encephalocele. Z Kinderchir Grenzgeb 28:347-351, 1979

8. Marín-Padilla M: Cephalic axial skeletal-neural dysraphic disorders: embryology and pathology. Can J Neurol Sci 18:153-169, 1991

9. Mealey J Jr, Dzenitis AJ, Hockey AA: The prognosis of encephaloceles. J Neurosurg 32:209-218, 1970

10. Simpson DA, David DJ, White J: Cephaloceles: treatment, 
outcome, and antenatal diagnosis. Neurosurgery 15:14-21, 1984

11. van Zalen-Sprock MM, van Vugt JM, van der Harten HJ, van Geijn HP: Cephalocele and cystic hygroma: diagnosis and differentiation in the first trimester of pregnancy with transvaginal sonography. Report of two cases. Ultrasound Obstet Gynecol 2:289-292, 1992

12. Winsor EJ, Brown BS: Prevalence and prenatal diagnosis of neural tube defects in Nova Scotia in 1980-84. CMAJ 135:1269-1273, 1986

\section{Disclosures}

The authors report no conflict of interest concerning the materials or methods used in this study or the findings specified in this paper.

\section{Author Contributions}

Conception and design: Gandhoke, Greene. Acquisition of data: all authors. Analysis and interpretation of data: all authors. Drafting the article: all authors. Critically revising the article: Gandhoke, Greene. Reviewed submitted version of manuscript: all authors. Approved the final version of the manuscript on behalf of all authors: Gandhoke. Administrative/technical/material support: Greene. Study supervision: Greene.

\section{Correspondence}

Gurpreet S. Gandhoke, University of Pittsburgh Medical Center, Neurological Surgery, 200 Lothrop St., Ste. B400, Pittsburgh, PA 15218. email: gandhokegs@upmc.edu. 